\title{
Reflets
}

Revue ontaroise d'intervention sociale et communautaire

\section{Les Ailes francophones : un pas vers l'autonomie des femmes}

\section{Pascale Houle et Magnolia Soutyrine}

Volume 9, numéro 2, automne 2003

Travail et mieux-être

URI : https://id.erudit.org/iderudit/011097ar

DOI : https://doi.org/10.7202/011097ar

Aller au sommaire du numéro

Éditeur(s)

Reflets : Revue ontaroise d'intervention sociale et communautaire

ISSN

1203-4576 (imprimé)

1712-8498 (numérique)

Découvrir la revue

Citer cet article

Houle, P. \& Soutyrine, M. (2003). Les Ailes francophones : un pas vers

l'autonomie des femmes. Reflets, 9(2), 199-206. https://doi.org/10.7202/011097ar

Tous droits réservés (C) Reflets : Revue ontaroise d'intervention sociale et communautaire, 2002
Ce document est protégé par la loi sur le droit d'auteur. L'utilisation des services d'Érudit (y compris la reproduction) est assujettie à sa politique d'utilisation que vous pouvez consulter en ligne.

https://apropos.erudit.org/fr/usagers/politique-dutilisation/ 


\title{
Les Ailes francophones : un pas vers l'autonomie des femmes
}

\author{
Pascale Houle, M.S.S., \\ Université d'Ottawa \\ Magnolia Soutyrine, \\ Agente de projet, Conseil de planification sociale d'Ottawa
}

\section{Historique}

En 1999, le Conseil de planification sociale d'Ottawa et ses partenaires ont effectué une recherche-intervention intitulée Le marché de l'emploi est-il travaillable? Les objectifs étaient d'identifier les barrières rencontrées par les femmes francophones dans leurs recherches d'emploi et de trouver des pistes de solutions pouvant favoriser leur intégration au marché du travail (Conseil de planification sociale d'Ottawa-Carleton 2000a). Un total de vingtsix femmes francophones immigrantes et canadiennes a participé à des entrevues de groupe. Trois groupes de discussion furent formés. Le premier était composé de mères d'origine canadienne, le second de mères néo-canadiennes et le troisième de femmes d'origine canadienne et néo-canadienne sans enfants (Conseil de planification sociale d'Ottawa-Carleton 2000a; Conseil de planification sociale d'Ottawa-Carleton 2000b). Les répondantes furent invitées à participer à une série de deux rencontres, la première traitant des obstacles rencontrés face au marché du travail et la seconde portant sur les solutions qu'elles envisageaient.

Les participantes furent impliquées dans toutes les étapes de la recherche. Une agente de projet a effectué l'analyse des résultats. 
Toutefois, ceux-ci furent présentés préalablement aux femmes de manière à vérifier s'ils reflétaient fidèlement ce qu'elles avaient discuté en groupe. Un colloque a eu lieu le $1^{\text {er }}$ mars 2000 dans le but de diffuser les résultats de la recherche et faire le lancement du rapport (Conseil de planification sociale d'Ottawa-Carleton 2000b). Enfin, nous avons invité les femmes à se joindre au comité directeur du projet afin de participer à l'élaboration des recommandations ainsi qu'à la diffusion du rapport de recherche Le marché de l'emploi est-il travaillable?

\section{Obstacles rencontrés par les femmes}

La réalisation de cette recherche a permis d'identifier différents obstacles dont ceux liés à l'emploi. Par ailleurs, les femmes ont aussi mentionné des obstacles culturels et évoqué d'autres problématiques vécues (Conseil de planification sociale d'OttawaCarleton 2000a; Conseil de planification sociale d'OttawaCarleton 2000b; Plante 2001; Plante 2000). Voici les principaux points soulevés par les participantes.

Au niveau des obstacles à l'emploi, on trouve:

- les exigences de formation spécialisée;

- les emplois à temps partiel, précaires ou mal rémunérés;

- les responsabilités familiales;

- la difficulté d'accès à une garderie et l'inflexibilité de l'horaire de travail avec les heures du service de garde;

- le problème de transport;

- la discrimination dans l'embauche par rapport au sexe et à l'âge;

- le manque de ressources d'aide à l'emploi;

- la recherche d'emploi longue et difficile et,

- l'isolement et le découragement dans la recherche d'emploi.

Au niveau des obstacles culturels, on trouve:

- l'absence de reconnaissance des acquis chez les femmes immigrantes (diplôme, expérience de travail, etc.); 
- le problème de langue, dont la méconnaissance de l'anglais;

- les attitudes discriminatoires des employeurs à l'endroit des minorités visibles (couleur de la peau, accent, etc.);

- la méconnaissance du marché du travail canadien et,

- l'information difficilement accessible.

Quant aux problématiques rencontrées, les femmes mentionnent:

- la pauvreté;

- l'isolement social;

- le manque de logements à prix abordable et,

- la faible estime de soi.

\section{En quoi consiste le groupe de Soutien communautaire à l'emploi? ${ }^{1}$}

Une des solutions retenue dans le rapport de recherche fut de créer un groupe de soutien pour les femmes francophones sans emploi de la région d'Ottawa. Un comité, formé uniquement de participantes et appuyé par une agente de projet, a déterminé les buts, les objectifs et la programmation des activités du groupe de soutien (Plante 2000). Ainsi, les Ailes francophones ${ }^{2}$ sont nées en juin 2000 (Plante 2001) suite à l'implication bénévole de femmes multi-ethniques désireuses d'améliorer la condition des femmes francophones de la région d'Ottawa et de promouvoir des valeurs telles que l'égalité, la justice sociale, le respect mutuel, l'autonomie et l'autodétermination. Composées d'une cinquantaine de femmes francophones immigrantes et canadiennes en recherche d'emploi, les Ailes francophones ont pour but d'offrir un service d'entraide et de support aux femmes d'expression française exposées à des problèmes liés à la pauvreté. Ce groupe permet aux femmes d'être accueillies et supportées dans leur démarche d'intégration au marché du travail. Une des caractéristiques qui le distingue des autres groupes existants dans la région, c'est qu'il se veut un forum où la voix des femmes peut être entendue. 


\section{Quelques résultats pour l'année 2002-2003}

Pendant l'année 2002-2003, le groupe a accueilli 59 femmes en recherche d'emploi. De ces femmes:

- $55 \%$ des femmes se sont trouvées un emploi;

- $17 \%$ ont commencé une formation;

- $20 \%$ sont en recherche d'emploi;

- $3 \%$ éprouvent des problèmes de santé;

- $5 \%$ sont en congé de maternité.

\section{Activités et services}

Le groupe de Soutien communautaire à l'emploi offre une gamme d'activités et de services aux femmes francophones dont un volet accueil et références, des rencontres thématiques, des repas communautaires, de l'information et de la formation (cours de langue, animation, apprentissage des outils informatiques), de l'accompagnement et un service téléphonique.

\section{Rencontres thématiques}

Des rencontres thématiques sont organisées une fois par mois selon le programme d'activités élaboré par le comité consultatif. Différents thèmes sont abordés tels le logement, l'assistance sociale, la recherche d'emploi, la sécurité alimentaire et les coopératives. Ces rencontres rejoignent une vingtaine de femmes qui bénéficient d'un support et d'une richesse d'informations. 


\section{Les Ailes francophones : incubateur de coopératives}

Des initiatives de développement économique communautaire ont pris leur envol dans le cadre des activités du groupe. En l'espace d'un an, quelques femmes ont mis sur pied deux coopératives de travail dans lesquelles participent des femmes canadiennes et immigrantes. En plus de constituer une source d'emploi, ces coopératives de travail permettent aux femmes de partager leurs idées, de trouver du soutien et de créer des liens de solidarité.

\section{Un projet innovateur : le marrainage dans le domaine de l'emploi}

L'idée du marrainage a été proposée par des femmes ayant participé à la recherche Le marché de l'emploi est-il travaillable? Des participantes à la recherche ont souligné que le marrainage contribuerait à briser l'isolement éprouvé par les femmes lorsqu'elles sont en recherche d'emploi. Les Ailes francophones ont créé un comité de marrainage afin de donner suite à la suggestion des femmes. Ce comité, composé de dix femmes francophones multi-ethniques de la ville d'Ottawa, a participé sur une base bénévole au développement des étapes du projet de marrainage en assistant à cinq rencontres d'une durée de deux heures trente chacune. Au cours de ces rencontres, plusieurs femmes ont donné leur nom pour devenir filleule et d'autres se sont montrées intéressées à s'engager à plus long terme soit en devenant marraine ou en s'impliquant bénévolement au niveau de l'administration du programme. Le développement du projet de marrainage a fait l'objet d'une demande de financement permettant la réalisation d'une recherche-action et d'un projetpilote d'une durée de six mois.

Le marrainage se définit par une relation d'entraide sur une base volontaire entre deux femmes francophones soit une marraine 
bénévole active sur le marché du travail et une filleule en recherche d'emploi. Cet accompagnement contribue à briser le sentiment d'isolement des femmes en recherche d'emploi par le biais du partage d'information, d'expériences et de connaissances dans une perspective de soutien entre les femmes. Le marrainage a pour but de favoriser l'intégration et le maintien en emploi des femmes francophones en milieu minoritaire pour qu'elles puissent améliorer leur situation économique. Les filleules sont accompagnées, supportées, conseillées et orientées dans leur démarche d'intégration au marché du travail et les marraines ont l'occasion de contribuer au cheminement professionnel de leur filleule en partageant leurs connaissances, leurs expériences ainsi que leur savoir-faire.

\section{Les débuts d'une cuisine collective}

Ce projet permet d'aborder la problématique de la sécurité alimentaire chez les femmes francophones multi-ethniques. Il contient deux volets: le premier consiste à développer un projet de recherche sur la cuisine collective qui visera à valider la cuisine collective comme méthode d'intervention et à démontrer son utilité au sein de la communauté francophone dans toute sa diversité. Le second volet consiste à mener un projet pilote sur une période de six mois dont le but est de concevoir un modèle de cuisine collective qui pourra être reproduit dans la communauté francophone ontarienne et d'élaborer ensuite un guide de référence.

\section{Composition du Conseil d'administration}

Les Ailes francophones offrent une place accueillante où les femmes peuvent réaliser leurs projets et aspirations. Les rapports entre les individus sont égalitaires et démocratiques. Ainsi, les décisions sont prises par les membres du groupe siégeant à 
différents comités dont le comité de réflexion, le comité consultatif et le comité directeur. Avec sa structure démocratique, le groupe donne l'opportunité aux femmes de s'exprimer et de s'affirmer.

\section{Conclusion}

Les Ailes francophones rejoignent plusieurs femmes francophones en milieu minoritaire. Celles-ci trouvent dans le groupe, non seulement un soutien dans leur recherche d'emploi, mais aussi un espace où elles peuvent échanger leurs idées, leurs craintes et proposer des solutions aux problèmes socio-économiques qu'elles vivent. Comme nous l'avons observé, la recherche d'emploi s'accompagne parfois de découragement et de solitude chez les femmes francophones et, plusieurs types d'obstacles limitent leur intégration au marché du travail. Cela explique la création du groupe de Soutien communautaire à l'emploi. Les femmes peuvent y trouver un accompagnement, un support en plus d'une foule d'activités et de services. Le développement de projets innovateurs comme le marrainage vise à favoriser l'insertion professionnelle et sociale de manière à ce que chaque personne trouve sa place. Comme le mentionne une femme du groupe:

je trouve que l'emploi représente la clé magique pour une bonne intégration dans le pays d'accueil. (...) Le problème de l'emploi est majeur chez les nouveaux Canadiens et pour arriver à freiner cette crise, il nous faut des groupes comme celui-ci, pour donner la voix aux femmes afin qu'elles puissent exprimer leurs besoins (Ailes francophones 2001).

Certes, l'intégration au marché du travail est une des principales formes d'inclusion dans la société puisqu'elle permet à l'individu de se distinguer par un rôle, une identité qui lui est propre et de se sentir utile en contribuant au développement de la collectivité. 


\section{Bibliographie}

AILES FRANCOPHONES (2001). "Ailes francophones: les témoignages», Notre Capitale Sociale, vol. 1, no. 1, 14-15.

CONSEIL DE PLANIFICATION SOCIALE D'OTTAWA-CARLETON (2003). Document descriptif. Projet soutien communautaire à l'emploi: «Les Ailes francophones», Ottawa, document inédit.

CONSEIL DE PLANIFICATION SOCIALE D'OTTAWA-CARLETON (2000a). Le marché de l'emploi est-il «travaillable»?, Ottawa.

CONSEIL DE PLANIFICATION SOCIALE D'OTTAWA-CARLETON (2000b). Le marché de l'emploi est-il «travaillable»?, Compte rendu du Colloque sur le soutien communautaire à l'emploi, Ottawa, avril 2000.

PLANTE, Nathalie C. (2001). «Soutien communautaire à l'emploi», Notre Capitale Sociale, vol. 1, no. $1,13$.

PLANTE, Nathalie C. (2000). «Des femmes d'Ottawa-Carleton s'expriment et se mobilisent», Les Cahiers de la femme/ Canadian Woman Studies, vol. 20, no 3, 127-128.

\section{Notes}

1. Les informations contenues dans cet article ont été tirées, en partie, du document descriptif sur le Soutien communautaire à l'emploi (Conseil de planification sociale d'Ottawa-Carleton 2003).

2. Ce groupe fut mis sur pied grâce à la contribution financière de laVille d'Ottawa et la collaboration de différents organismes communautaires. 